OPEN ACCESS

Edited by:

Lixing Huang,

Jimei University, China

Reviewed by:

Jianjun Wang,

Jiangsu University, China

Li Mofei,

Chinese Academy of Sciences, China

*Correspondence:

Chuangfu Chen

chuangfu_chen@163.com

Yong Wang

577674101@qq.com

tThese authors have contributed equally to this work

Specialty section: This article was submitted to Veterinary Infectious Diseases,

a section of the journal

Frontiers in Veterinary Science

Received: 17 December 2020 Accepted: 11 February 2021

Published: 16 March 2021

Citation:

Yi J, Wang $Y$, Zhang $H$, Deng $X, X i J$, $L i H$, Yang $N$, Ma Z, Wang $Y$ and Chen C (2021) Interferon-Inducible Transmembrane Protein 3-Containing Exosome as a New Carrier for the Cell-to-Cell Transmission of Anti-Brucella Activity.

Front. Vet. Sci. 8:642968

doi: 10.3389/fvets.2021.642968

\section{Interferon-Inducible Transmembrane Protein 3-Containing Exosome as a New Carrier for the Cell-to-Cell Transmission of Anti-Brucella Activity}

\author{
Jihai $\mathrm{Yi}^{1}{ }^{1,2+}$, Yueli Wang ${ }^{1+}$, Huan Zhang ${ }^{1}$, Xiaoyu Deng ${ }^{1}$, Jing Xi ${ }^{1}$, Honghuan $\mathrm{Li}^{1}$, \\ Ningning Yang ${ }^{1}$, Zhongchen Ma ${ }^{1}$, Yong Wang ${ }^{1 *}$ and Chuangfu Chen ${ }^{1 *}$ \\ ${ }^{1}$ College of Animal Science and Technology, Shihezi University, Shihezi, China, ${ }^{2}$ Key Laboratory of Control and Prevention of \\ Animal Disease, Xinjiang Production \& Construction Corps, Shihezi, China
}

Exosomes are small extracellular vesicles that are released from cells and that function in intercellular communication. Recently, interferon-inducible transmembrane protein 3 (IFITM3) has been identified as a highly effective anti-intracellular pathogen protein that can inhibit the invasion of a wide range of pathogenic microorganisms. However, whether Brucella infection induces secretion of exosomes and whether these exosomes contain IFITM3 protein remain unknown. Here, we focused on the immune function of extracellular IFITM3 protein in the process of Brucella infection. This study is the first to show that Brucella melitensis strain M5 (Brucella M5) can stimulate macrophages to secrete large amounts of exosomes. Most importantly, we identified exosomes from Brucella M5-infected cells that were rich in molecules of IFITM3, and these exosomes could transmit the IFITM3 from one cell to another, thereby effectively inhibiting the intracellular survival of Brucella. Moreover, immunization with exosomes carrying IFITM3 decreased mouse spleen tissue damage and spleen colony forming unit (CFU), leading to the establishment of an anti-Brucella state in mice. In conclusion, our findings provide new insights into the anti-Brucella mechanism of IFITM3-containg exosomes, thus providing a theoretical foundation for systematic elaboration of the mechanisms of Brucella infection and host immunity. The results provide new ideas for the development of candidate vaccines for Brucella.

Keywords: Brucella, exosome, label-free proteomic analysis, IFITM3, immunity, intracellular survival

\section{INTRODUCTION}

Brucella is a genus of Gram-negative facultative intracellular bacteria that cause serious damage to domestic animals and humans $(1,2)$. In animals, brucellosis predominantly causes chronic epididymitis in males and abortions and sterility in females, resulting in serious economic losses in livestock husbandry production (3). In humans, brucellosis causes joint pain, undulant fever, and fatigue. Studies have confirmed that Brucella has evolved a high-level immune escape strategy so that the host immune system cannot completely eradicate the bacteria; the pathogen can thus eventually establish a chronic infection (4). At present, it is believed that Brucella infection can stimulate the innate immunity of the body (5). Macrophages, as an important component of the innate immune system, are the 
key cells in defense against pathogen invasion (6). Studies have shown that the activation of macrophages plays an important role in clearing and controlling Brucella; $90 \%$ of the invading Brucella can be killed by eventual fusion with lysosomes (7). Therefore, understanding the relationship between Brucella infection and innate immunity and the interaction among immune cells is of great significance for controlling the spread of brucellosis.

Exosomes are small membrane-bound vesicles with diameters ranging from 30 to $120 \mathrm{~nm}$. Exosomes are formed in the endosomal compartment by inward budding of endosomal membranes that are produced in late endosomal intracellular multivesicular bodies (8) (MVBs). After the fusion of MVBs with the plasma membrane, pools of exosomes accumulated in MVBs are released into the extracellular space (9). Those small vesicles can carry proteins, nucleic acids, and lipids to neighboring cells and thus modulate many biological processes such as cell growth, immune regulation, and cell migration (10-12). At present, research on the role of exosomes in the host immune response mainly focuses on tumor immunity, autoimmunity, and viral infection, while there are few studies on the role of exosomes in bacterial infections (especially intracellular bacteria). A recent study reported the protein profiles of extracellular vesicles-derived macrophages with Mycobacterium tuberculosis (Mtb) analyzed by label-free liquid chromatographymass spectrometry (LC-MS)/MS quantitation technology. A total of 287 protein groups were identified in these vesicles, including host immune proteins and tuberculosis virulencerelated proteins (13), and the host proteins played an important role in innate immunity (14). However, it is unknown whether Brucella, an intracellular bacterium, can also induce macrophages to produce exosomes.

Interferon-inducible transmembrane protein 3 (IFITM3) belongs to the family of IFN-stimulated genes (ISGs) (15) that are key antiviral effectors of the host innate immune system (16). Research has indicated that IFITM3 is also a major immune molecule in the host defense against Mtb infection (17). The reduction of $\mathrm{pH}$ value in lysosomes of host cells mediated by IFITM3 can effectively inhibit the intracellular proliferation of Mtb, suggesting that IFITM3 not only participates in antiviral immunity but also plays a crucial role in inhibiting the survival of intracellular bacteria $(17,18)$. The results from the study of Zhu have shown that IFITM3-containing exosomes were present in the extracellular microenvironment, and that study also determined that these exosomes could transmit the IFITM3mediated antiviral activity from Dengue virus infected-cells to uninfected cells (19), revealing a new mode of antiviral defense. However, in the process of Brucella infection, whether IFITM3containing exosomes have notable anti-intracellular bacterial activity in hosts is unknown.

This study is the first to extract and identify exosomes from the extracellular environment of Brucella M5-infected macrophages. Label-free proteomics analysis of such exosomes indicated that immune molecules of IFITM3 were enriched in the exosomes. Subsequently, we focused our investigation on the function of the IFITM3-laden exosomes in vivo and in vitro.

\section{MATERIALS AND METHODS}

\section{Cells, Brucella Strains, and Mice}

Brucella M5 was provided by the Center for Chinese Disease Prevention and Control (Beijing, China). Brucella M5 was cultured in tryptone soya agar (TSA) or tryptone soya broth (TAB) at $37^{\circ} \mathrm{C}$. Macrophages RAW264.7 were obtained from the Type Culture Collection of the Chinese Academy of Sciences (Shanghai, China). The macrophages were cultured in a $5 \% \mathrm{CO}_{2}$ atmosphere in DMEM containing 10\% exosome-depleted fetal bovine serum (FBS). Six-week-old BALB/c mice were purchased from the Xinjiang Medical University. All animal care in this experiment was performed in compliance with institutional animal care guidelines and relevant laws.

\section{Brucella M5 Infection of Macrophages RAW264.7}

Macrophages RAW264.7 were infected with Brucella M5 for $1 \mathrm{~h}$; the multiplicity of infection (MOI; bacteria:cell) was 100:1. Cells were incubated for $60 \mathrm{~min}$. The macrophages were then washed three times with phosphate-buffered saline (PBS). Next, gentamicin $(25 \mu \mathrm{g} / \mathrm{ml})$ was added to the cell culture medium and incubated for $45 \mathrm{~min}$ to kill bacteria outside of the cells. The culture medium was discarded and replaced. After $24 \mathrm{~h}$ post-Brucella infection, the supernatants were collected from each plate.

\section{Isolation and Identification of Exosomes}

Exosomes from each of the culture supernatants above were obtained by differential centrifugation. Briefly, cell supernatants were centrifuged at $1,000 \mathrm{~g}$ for $5 \mathrm{~min}, 5,000 \mathrm{~g}$ for $5 \mathrm{~min}, 14,000$ $g$ for $30 \mathrm{~min}$, and $120,000 \mathrm{~g}$ for $1 \mathrm{~h}$, followed by two wash with PBS buffer and purification to eliminate contaminating proteins by centrifugation at $120,000 \mathrm{~g}$ for $1 \mathrm{~h}$. The purified exosomes were identified by nanoparticle tracking analysis (NTA), transmission electron microscopy (TEM), and Western blotting as described by Mitchell (20). The total protein concentration of exosomes was measured using the BCA Assay Kit (Pierce, Rochford, IL). Before the experiment, all isolated exosomes were stored at $-80^{\circ} \mathrm{C}$.

\section{Label-Free Analysis}

In this experiment, the identified exosomes were sent to Applied Protein Technology Co., Ltd (Shanghai., China) for label-free quantitative proteomics analysis. The exosome samples were divided into two groups: one group comprised of exosomes released from normal macrophages RAW264.7 (uninfected group), and the other contained exosomes released from Brucella-infected macrophages RAW264.7 (infected group), with three replicates per group.

\section{Interferon-Inducible Transmembrane Protein 3 Interference Vector and Identification of Stable Expression Cell Lines}

The 293FT cells and macrophages RAW264.7 were transfected with lentiviral packaging plasmids LV1-IFITM3 (siIFITM3) designed by GenePharma (Shanghai, China) and helper plasmids 
TABLE 1 | Lentivirus packaging system.

\begin{tabular}{llc}
\hline Group & Reagent & Dosage $(\boldsymbol{\mu l})$ \\
\hline A & Buffer A & 250 \\
B & LV1-IFITM3 & 6 \\
& PRSV-Rev & 3 \\
& PMDL & 3 \\
& PCMV-VSVG & 3 \\
& dd $\mathrm{H}_{2} \mathrm{O}$ & Supplement to 250
\end{tabular}

TABLE 2 | RNA transcription system of reverse transcription-PCR (RT-PCR).

\begin{tabular}{lllc}
\hline Gene & Primer name & Primer $\left(\mathbf{5}^{\prime} \rightarrow \mathbf{3}^{\prime}\right)$ & Tm $\left({ }^{\circ} \mathbf{C}\right)$ \\
\hline IFITM3 & IFITM3-F & GTC TCGCTCCTGGAAGAT GGTG & 61 \\
& IFITM3-R & CATTGCAG TGGCAAAGT GGAG & \\
Reference gene & GAPDH-F & GGTGAAGGTCGGTGAACG & 58 \\
& GAPDH-R & CTCGCTCCTGGAAGATGGTG & \\
& & &
\end{tabular}

(PRSV-Rev, pMDL, and PCMV-VSVG) by Lipofectamine 2000 (Invitrogen, OH, USA). The ratios of plasmids are listed in Table 1. All of the plasmids and macrophages were mixed thoroughly and cultured at $37^{\circ} \mathrm{C}$ in a $5 \% \quad \mathrm{CO}_{2}$ incubator. IFITM3 stable interference cells (siIFITM3-Mø) were generated. After incubation for $48 \mathrm{~h}$, the cell lines were identified using fluorescence microscopy (Nikon, Japan) and RT-PCR (Table 2).

\section{Western Blot Assays}

The different pretreated cells were lysed by high-efficiency RIPA Lysate (Solarbio, China) and boiled for $10 \mathrm{~min}$ at $100^{\circ} \mathrm{C}$ in SDS buffer. The total proteins were separated using SDS-PAGE and then transferred onto nitrocellulose membranes (Millipore, MA, USA). The membranes were blocked with 5\% non-fat milk (Thermo Scientific, MA, USA) and probed with rabbit antiphospho-IFITM3 (dilution 1:2,000, EterLife, China) for $1 \mathrm{~h}$ at $37^{\circ} \mathrm{C}$. The membranes were incubated with HRP-conjugated mouse anti-rabbit IgG (dilution 1:5,000, ZSGB-BIO, China) for $1 \mathrm{~h}$ at $37^{\circ} \mathrm{C}$. Finally, membranes were washed and stained using the HRP-DAB kit (ZSGB-BIO, China). Protein synthesis was analyzed by an enhanced chemiluminescence system.

\section{Real-Time PCR}

Total RNA was isolated from siIFITM3-Mø cells and reverse transcribed to cDNA, with GAPDH as a reference gene. RT-PCR was performed with SYBR-Green (Roche, Switzerland) along with the primers listed in Table 2. Data were analyzed using the comparative $2^{-\Delta \Delta \mathrm{Ct}}$ method.

\section{Colony Forming Units (CFU) and Cytokine Production Assay}

Exosomes released from the different pretreated cells were incubated with siIFITM3-M cells for $8 \mathrm{~h}$ and then infected with Brucella M5 (the infection method was as described above) and incubated for $4,12,24$, and $48 \mathrm{~h}$ post-infection. Then, the cells were lysed with $0.5 \mathrm{ml}$ of $0.2 \%$ Tween 20 for $15-30 \mathrm{~min}$ on ice, followed by rinsing of each well with $0.5 \mathrm{ml}$ of PBS.
Viable bacteria were quantified by serial dilution in sterile PBS and plating on TSA, and then were incubated at $37^{\circ} \mathrm{C}$ for $72 \mathrm{~h}$ followed by colony counting. And the levels of IFN- $\alpha$ and IFN$\beta$ in the supernatants were measured with an ELISA Quantikine Mouse kit (R\&D Systems, MN, USA). The data were obtained from three independent experiments.

\section{Immunization of Mice With Exosomes}

A total of four immunization programs were formulated: exosomes from uninfected macrophages RAW264.7 (Exo), exosomes from Brucella M5-infected macrophages RAW264.7 (Exo-M5-IFITM3), exosomes from Brucella M5 infected siIFITM3-Mø cells (Exo-M5-siIFITM3), and a PBS group. Each immunization program included three mice, and each of the mice was immunized via the tail vein route with a final injection volume of $100 \mu \mathrm{l}(0.3 \mu \mathrm{g} / \mu \mathrm{l})$ (the injection dose was referred to Cheng and Schorey (21). Immunization was repeated three times at 5-day intervals.

\section{Brucella Challenge}

Thirty-five days following the final vaccination with exosomes, each group of mice was challenged with Brucella M5 by subcutaneous injection $\left(1 \times 10^{6} \mathrm{CFU} /\right.$ mouse in $100 \mu \mathrm{l}$ of PBS). After 1, 3, 5, and 7 weeks post-infection, three Brucella-infected mice were humanely sacrificed to remove and weigh the spleens. The spleen was put into a centrifuge tube containing PBS buffer, $1 \mathrm{ml}$ of $0.2 \%$ Triton X-100, and several small steel balls, and then homogenized in a homogenizer. Then, $100 \mu$ l of the homogenate was diluted in sterile saline and plated onto TSA. The plates were incubated at $37^{\circ} \mathrm{C}$, and bacterial colony counts were expressed as $\log _{10} \mathrm{CFU}$ per spleen.

\section{RESULTS}

\section{Characterization of Exosomes Secreted From Brucella-Infected Macrophages RAW264.7}

Exosomes released from Brucella M5-infected cells were analyzed by EM, NTA, and Western blotting. The results showed that these exosomes had a spherical cup-shaped appearance, with an average size of 40-120 nm (Figures 1A-C). In addition, comparison of macrophages RAW264.7 lysates with exosomal preparations indicated the presence of multiple specific exosome markers CD63 and Tsg101 (Figure 1D).

\section{Label-Free Proteomic Analysis of Exosomes Secreted From Brucella-Infected Macrophages RAW264.7}

The aim of the analysis was to comprehensively explore the components of exosome-derived macrophages RAW264.7. Label-free quantitation technology was used to analyze the protein profiles of exosomes secreted from macrophages with or without Brucella infection. A total of 1,236 proteins were identified (Figure 2A), including 1,156 proteins derived from host cells and 80 proteins derived from Brucella M5. Among the 1,156 proteins, 89 proteins were found to be upregulated 
A

Cell culture supernatant

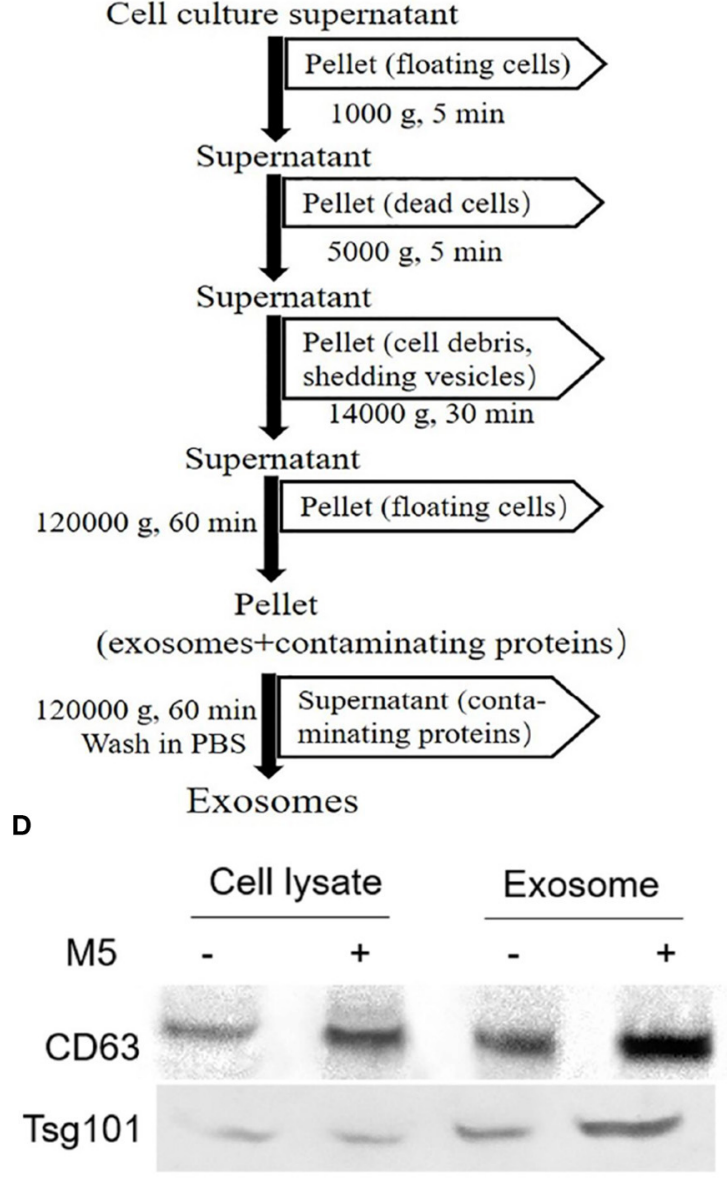

B

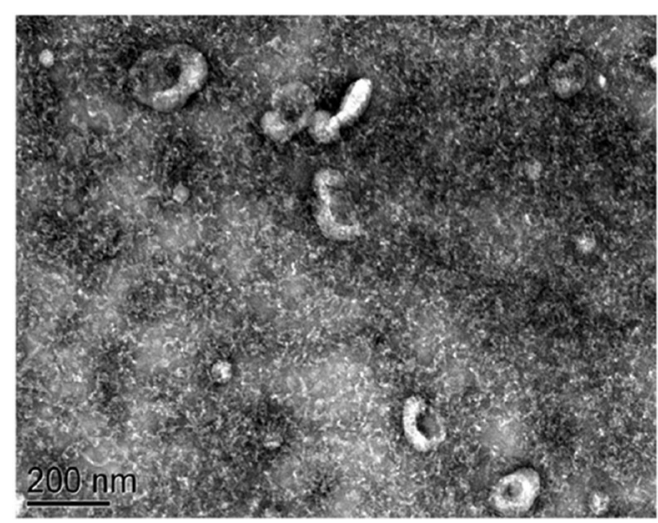

C



FIGURE 1 | Purification and identification of exosomes secreted from Brucella M5-infected macrophages. (A) Method of the isolation and purification of exosomes from cell culture supernatant based on differential centrifugation. The purified exosomes secreted from Brucella-infected macrophages were analyzed by transmission electron microscopy (TEM). (B) The exosomes secreted from Brucella-infected macrophages size distributions were tested by nanoparticle tracking analysis (NTA) (C). (D) Western blotting was used to identify exosomal markers (CD63, Tsg101) in exosomes (5 $\mu \mathrm{g} /$ well) derived from uninfected or Brucella M5-infected macrophages and in the corresponding whole cell lysates.

(Figures 2B,C), including interferon-induced transmembrane protein (IFITM3), lysozyme C-2 (Lyz2), and CD14 (Table 3). The functions of these proteins involved host immunity. This study focused on the biological functions of IFITM3. Based on the above results, we further explored the functions of IFITM3 in Brucella infection and host immunity.

\section{Silencing the IFITM3 Gene Was Beneficial to the Intracellular Survival of Brucella M5}

To understand the role of endogenous IFITM3 in the interaction between Brucella and host macrophages, we first utilized siRNA technology to successfully silence the expression of the IFITM3 gene in macrophages RAW264.7 (Figures 3A,B). After infection with Brucella M5 at different time points, Western blot analysis showed that Brucella M5 infection could induce the activation of IFITM3 in a time-dependent manner, reaching the highest value at $24 \mathrm{~h}$. Moreover, the expression of IFITM3 was inhibited in siIFITM3-Mø cells. Adding GW4869, a specific inhibitor of exosome release (22), or an empty interference vector did not affect IFITM3 expression (Figure 3D). IFITM3silenced macrophages infected with Brucella were lysed, and the CFU results showed that compared with the PBS control group, silencing the IFITM3 gene significantly promoted the intracellular survival of Brucella M5 $(p<0.05)$ in a timedependent manner. However, the empty interference vector did not affect the intracellular survival of Brucella M5 (Figure 3C). These results suggest that IFITM3 expression in macrophages contributes to host cell resistance to Brucella infection.

\section{IFITM3 Can Be Released to Extracellular Space via the Exosomal Pathway}

To determine whether exosomes derived from Brucella M5-infected macrophages RAW264.7 were able to transfer immunocompetent IFITM3 molecules to the extracellular 

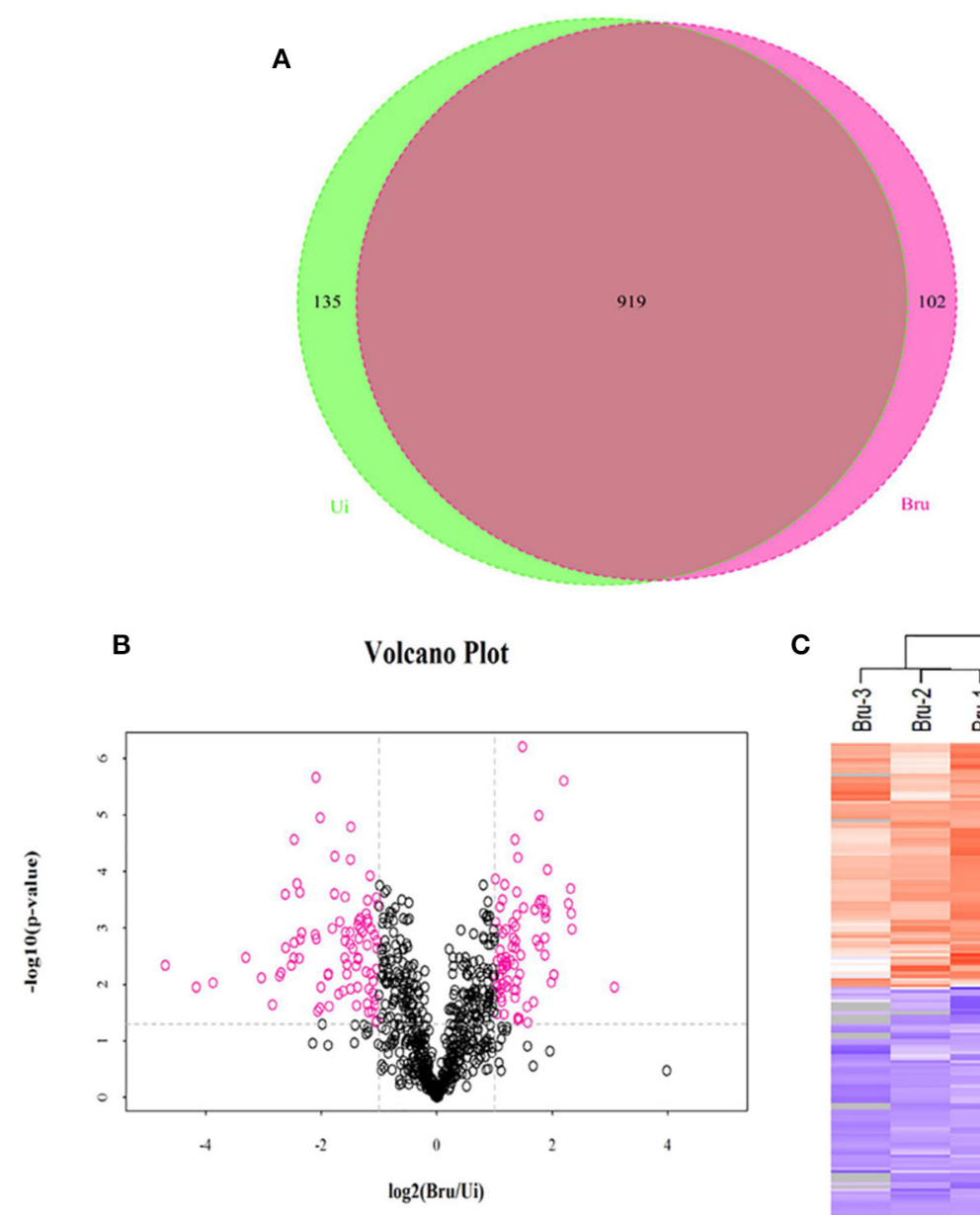

C


FIGURE 2 | Proteomic profiling of exosomes derived from uninfected or Brucella M5-infected macrophages. (A) Venn diagram showing the overlap between exosome derived from Brucella M5-infected cells and uninfected cells. (B) Volcano plot and Heatmap (C) showing the differential protein expression of these two groups.

space, we extracted exosomes in cell culture supernatants at different time points of Brucella M5 infection. The results showed that exosomes derived from macrophages RAW264.7 contained a high abundance of IFITM3 in a time-dependent manner, reaching a maximum at $24 \mathrm{~h}$ post-infection (Figure $4 \mathrm{~A}$ ). However, as shown in Figure 4B, there was no IFITM3 protein inside exosomes derived from Brucella-infected siIFITM3-Mø cells. Furthermore, different multiplicity of infection (MOI) levels affected the ability of exosomes to load IFITM3, reaching the highest loading capacity with an MOI of 100 (number of bacteria: number of cells $=100: 1$ ) (Figure 4C). Taken together, our data demonstrate that IFITM3 can be exported from macrophages by the exosomal pathway.

\section{IFITM3-Containing Exosomes Are Internalized by Macrophages}

The finding above, that exosomes derived from Brucella-infected macrophages carried a high abundance of IFITM3 and were released into the extracellular space, prompted us to explore whether the IFITM3-laden exosomes were internalized by other host macrophages in the immune system. If true, this would mean that the anti-Brucella activity could be transferred to the target macrophages. In this study, we investigated such a possibility by incubating macrophages RAW264.7 pretreated with exosome inhibitor GW4869 with exosomes collected from Brucella M5-infected cells (Exo-M5-IFITM3), Brucella-infected siIFITM3-Mø cells (Exo-M5-siIFITM3), or normal cells (Exo). As shown in Figure 5A, a significant increase in the abundance of IFITM3 was observed in the target macrophages incubated with Exo-M5-IFITM3 $(30 \mu \mathrm{g} / \mathrm{ml})$, and the maximum value was reached at $12 \mathrm{~h}$ post-incubation. However, the incubation of ExoM5-siIFITM3 and Exo hardly affected the protein abundance of IFITM3 inside target macrophages. In addition, detection of IFITM3 proteins in the lysates of siIFITM3-Mø cells treated with Exo-M5-IFITM3 for $12 \mathrm{~h}$, as determined by Western blotting, was dose-dependent, reaching the highest value at $30 \mu \mathrm{g} / \mathrm{ml}$. Interestingly, $30 \mu \mathrm{g} / \mathrm{ml}$ of Exo-M5-siIFITM3 did not increase the abundance of IFITM3 protein in the siIFITM3Mø cells (Figure 5C). Furthermore, in order to confirm that the increased IFITM3 protein abundance in the recipient 
TABLE 3 | List of major protein expression differences in exosomes from host cells.

\begin{tabular}{|c|c|c|c|c|c|}
\hline Protein ID & Protein name & Gene name & Bru/Ui & $T$-test $p$-value & state \\
\hline Q9CQW9 & Interferon-induced transmembrane protein 3 & IFITM3 & 4.44 & 0.0037 & Up \\
\hline P08905 & Lysozyme C-2 & Lyz2 & 3.30 & 0.0016 & Up \\
\hline P14211 & Calreticulin (MHC-I) & Calr & 2.61 & 0.0442 & Up \\
\hline Q9Z0M5 & Lysosomal acid lipase/cholesteryl ester hydrolase & Lipa & 2.57 & 0.0022 & Up \\
\hline Q61937 & Nucleophosmin & Npm1 & 2.56 & 0.0027 & Up \\
\hline P80316 & T-complex protein 1 subunit epsilon & Cct5 & 2.26 & 0.0001 & Up \\
\hline P10810 & Monocyte differentiation antigen CD14 & CD14 & 2.23 & 0.0119 & Up \\
\hline Q9Z0X1 & Apoptosis-inducing factor 1 , mitochondrial & Aifm1 & 2.21 & 0.0045 & Up \\
\hline О09159 & Lysosomal alpha-mannosidase & Man2b1 & 2.19 & 0.0003 & Up \\
\hline P15379 & CD44 antigen & CD44 & 2.16 & 0.1230 & Up \\
\hline P26151 & High affinity immunoglobulin gamma Fc receptor I & Fcgr1 & 2.12 & 0.0052 & Up \\
\hline P41731 & CD63 antigen & Cd63 & 2.08 & 0.0329 & Up \\
\hline Q8ClH5 & phospholipase C-gamma (1) & Plc $\gamma 2$ & 2.01 & 0.0112 & Up \\
\hline Q99MN9 & Propionyl-CoA carboxylase beta chain, mitochondrial & Pccb & 2.00 & 0.0378 & Up \\
\hline Q8VBV7 & COP9 signalosome complex subunit 8 & Cops8 & 0.49 & 0.0011 & Down \\
\hline P40237 & CD82 antigen & Cd82 & 0.49 & 0.0180 & Down \\
\hline P51863 & V-type proton ATPase subunit d 1 & Atp6v0d1 & 0.45 & 0.0298 & Down \\
\hline P57746 & V-type proton ATPase subunit D & Atp6v1d & 0.43 & 0.0003 & Down \\
\hline P61957 & Small ubiquitin-related modifier 2 & Sumo2 & 0.47 & 0.0092 & Down \\
\hline Q9CZ04 & COP9 signalosome complex subunit 7a & Cops7a & 0.24 & 0.0111 & Down \\
\hline Q03265 & ATP synthase subunit alpha, mitochondrial & Atp5f1a & 0.38 & 0.0035 & Down \\
\hline
\end{tabular}

macrophages was due to IFITM3-laden exosomes instead of a stimulated expression of endogenous IFITM3 genes in the recipient cells, we measured the IFITM3 mRNA transcription levels in normal macrophages pretreated with Exo-M5-siIFITM3, Exo-M5-siNC, or Exo-M5-IFITM3 via RT-PCR (Figure 5B). The results showed that exosomes carrying or not carrying IFITM3 did not cause an increase of endogenous IFITM3 in the receptor macrophages. Taken together, these results demonstrated that exosomes carrying IFITM3 protein can be internalized by recipient macrophages, thereby resulting in an increase in the abundance of the IFITM3 protein.

\section{The Anti-Brucella Activity of Internalized IFITM3-Containing Exosomes}

To investigate whether IFITM3-containing exosomes (Exo-M5IFITM3) internalized by recipient macrophages could affect intracellular survival of Brucella, siFITM3-Mø cells were cultured in DMEM containing 10\% exosome-depleted FBS and exposed to $30 \mu \mathrm{g}$ of purified Exo-M5-IFITM3 for $12 \mathrm{~h}$. After Brucella M5 infected the exosome-treated cells, CFU counts were performed at different time points post-infection. As shown in Figure 6A, compared with the PBS control group, the number of intracellular bacteria in recipient cells pretreated with Exo-M5-IFITM3 or Exo-M5-siNC significantly decreased after $4,12,24$, and $48 \mathrm{~h}$ post-Brucella infection $(p<0.05)$, but the Exo-M5-siIFITM3 and PBS groups had no effect on the intracellular replication of Brucella M5. In order to verify the relationship between the release of type 1 interferon and the expression of IFITM3, ELISA results showed that
Brucella M5 could induce macrophages to release IFN- $\beta$ and IFN- $\alpha$; however, IFITM3-exosomes treatment did not affect the expression of IFN- $\alpha$ (Figure 6B) and IFN- $\beta$ (Figure 6C). Taken together, our data indicated exosome delivery of IFITM3 to recipient macrophages, thereby effectively inhibiting the intracellular survival of Brucella and conferring resistance to Brucella persistent infection. Interestingly, IFITM3-containing exosomes mediated the decrease in intracellular replication of Brucella was not due to the release of non-specific type I interferon.

\section{Interferon-Inducible Transmembrane Protein 3-Containing Exosomes Protected Mice Against Brucella M5 Infection}

To determine whether IFIMT3-containing exosomes could boost protective activity against Brucella infection, mice were given a dose of $1 \times 10^{6} \mathrm{CFU}$ with Brucella M5 after the final exosome immunization (35 days). Then, 1, 3, 5, and 7 weeks later, each group of three mice was sacrificed, and then the spleen index (spleen weight/body weight) and spleen CFU were calculated. As shown in Figure 7A, in the Exo-M5-IFITM3 group, the spleen index showed a significant increase compared with the Exo-M5siIFITM3 group at 3 weeks of infection but was still lower than that of the PBS control group at 1,3 , and 5 weeks of infection ( $p$ $<0.05)$. The CFU test showed that the Exo-M5-IFITM3 group had a significantly higher spleen bacterial load compared to the Exo-M5-siIFITM3 group at 3, 5, and 7 weeks of infection, but was still lower than that of the PBS control group at 3 and 5 weeks of infection $(p<0.05)$ (Figure 7B). In addition, immunization 
A
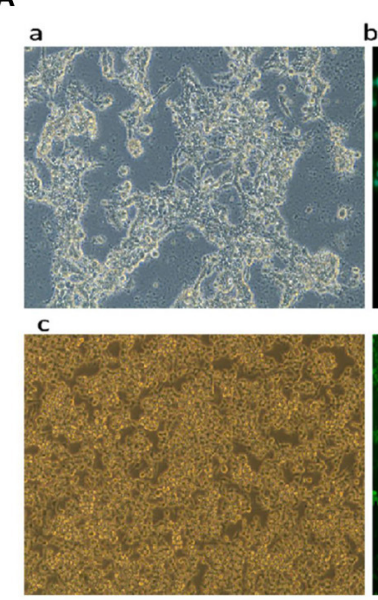

C

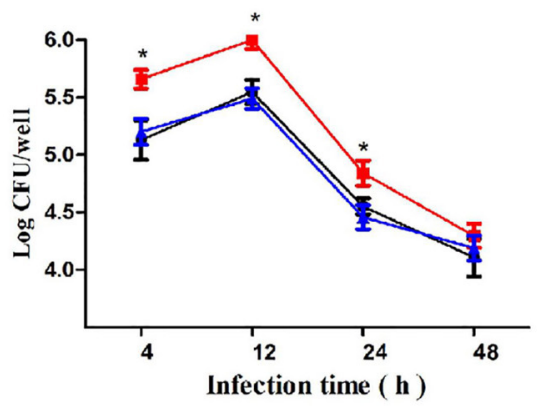

b

d
B

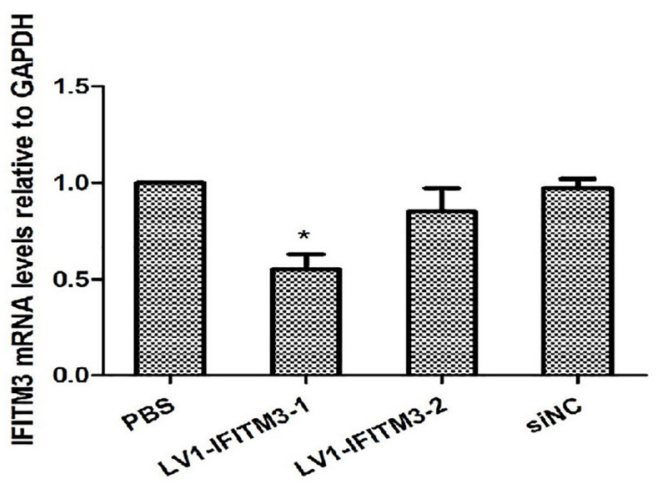

D


FIGURE 3 | IFITM3 protein is required for the establishment of host immune response against Brucella. (A) LV1-IFITM3 packaging and infection process. (a): 293T cells; (b): Lentivirus package; (c): RAW264.7 cells; (d): Lentivirus transfection. (B) RT-PCR analysis of relative mRNA expression of IFITM3 in macrophages infected with the siRNA-IFITM3 lentivirus vectors. The $2^{-\Delta \Delta C t}$ method was used to calculate relative gene expression. (C) Brucella intracellular growth was determined after 4 , 12, 24, and $48 \mathrm{~h}$ post-infection by CFU test. (D) Macrophages pretreated with IFITM3 siRNA, siNC, or GW4869 were infected with Brucella M5 and were lysed after $24 \mathrm{~h}$ post-infection. The IFITM3 in cell lysate was analyzed by Western blotting. All treatments were repeated three times with $n=3 /$ time point. Statistical significance is indicated by ${ }^{*} p<0.05$.

of exosomes from uninfected macrophages RAW264.7 (Exo group) did not decrease the spleen index or spleen CFU. Thus, these results indicated that exosomes released from Brucella M5-infected macrophages could reduce Brucella-induced spleen tissue damage and provide protective activity against Brucella infection. IFITM3 contained in exosomes might be a key cellular restriction factor for Brucella infection.

\section{DISCUSSION}

Interferon-inducible transmembrane protein 3 is an efficacious restriction factor with broad-spectrum antiviral activity in host cells (23). However, an important finding of our present study, that antiviral molecules of IFITM3 can be transferred intercellularly from Brucella-infected macrophages to uninfected neighbors by extracellular exosomes and thereby confer resistance to Brucella persistent infection, provides new insights into the functional significance of antimicrobial induced by IFITM3. Our verification of this ability of IFITM3-laden exosomes may provide a foundation for further elucidating the molecular mechanism of Brucella infection and host innate immunity, eventually leading to the development of new candidate vaccines against Brucella.

Although IFITM3 is widely used in antiviral research, some studies had found that IFITM3 expression could enhance endosomal acidification in Mtb-infected monocytic cells, thereby directly inhibiting bacterial pathogen growth and implying that IFITM3 may be involved in the immune response against intracellular bacteria $(24,25)$. Several other intracellular pathogens, such as Salmonella enterica or Yersinia pestis, also depend on phagolysosome acidification arrest for survival and reproduction in host cells (26). Thus, IFITM3 may have a similar biological function during Brucella infection. This study found that Brucella M5 can indeed induce macrophages to express IFITM3 in a time-dependent manner. Silencing the IFITM3 gene was beneficial to the intracellular survival of Brucella M5. During our experiment, the interference efficiency of IFITM3 was always about $50 \%$, which might be due to the type of host cell. But fortunately, the interference efficiency of $50 \%$ could also significantly affect the survival of Brucella in vivo and in vitro. It also indirectly indicated that IFITM3 protein played an important role in the process of resistance to bacterial 


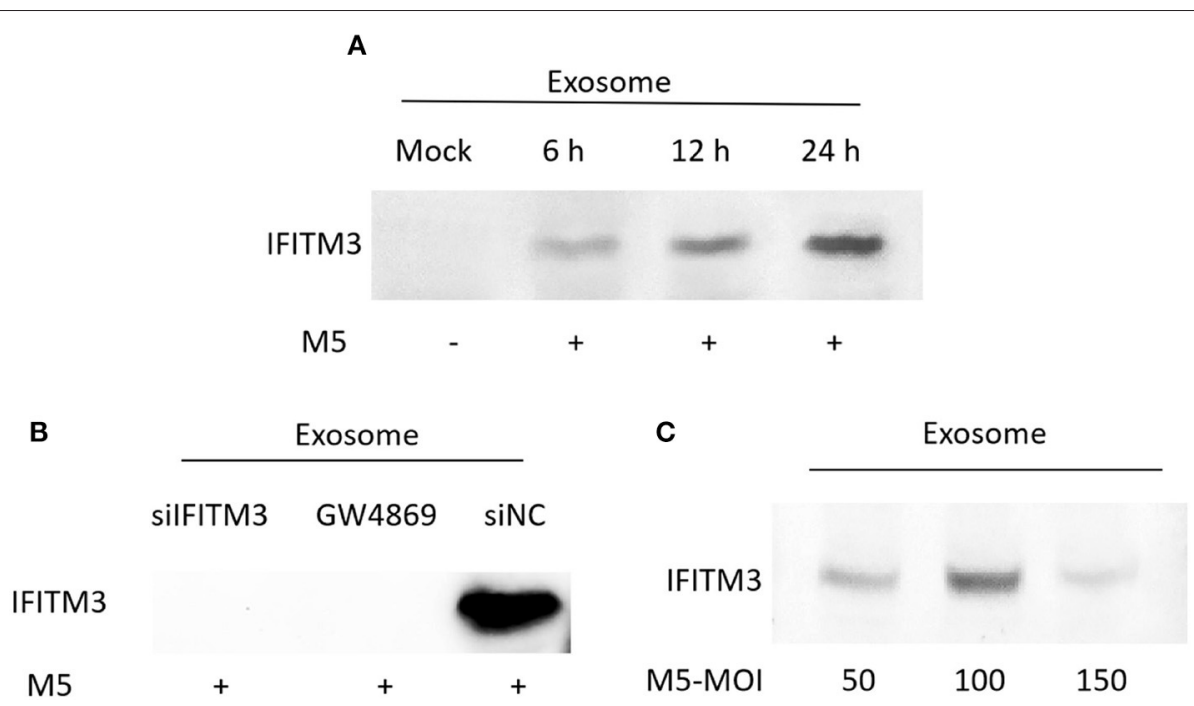

FIGURE 4 | Identification of IFITM3 in exosomes secreted from Brucella M5-infected cells. (A) Macrophages were infected with Brucella M5 for 6, 12, and 24 h, and exosomes in the cell culture media were extracted. Western blot analysis of IFITM3 protein in exosome. (B) Macrophages pretreated with IFITM3 siRNA, siNC, or GW4869 were infected with Brucella M5, and exosomes derived from each group were extracted. Western blot analysis of IFITM3 protein in exosome. (C) Macrophages were infected with Brucella M5 at different MOI, and exosomes in this cell culture medium were extracted. Western blot analysis of IFITM3 protein in exosomes.

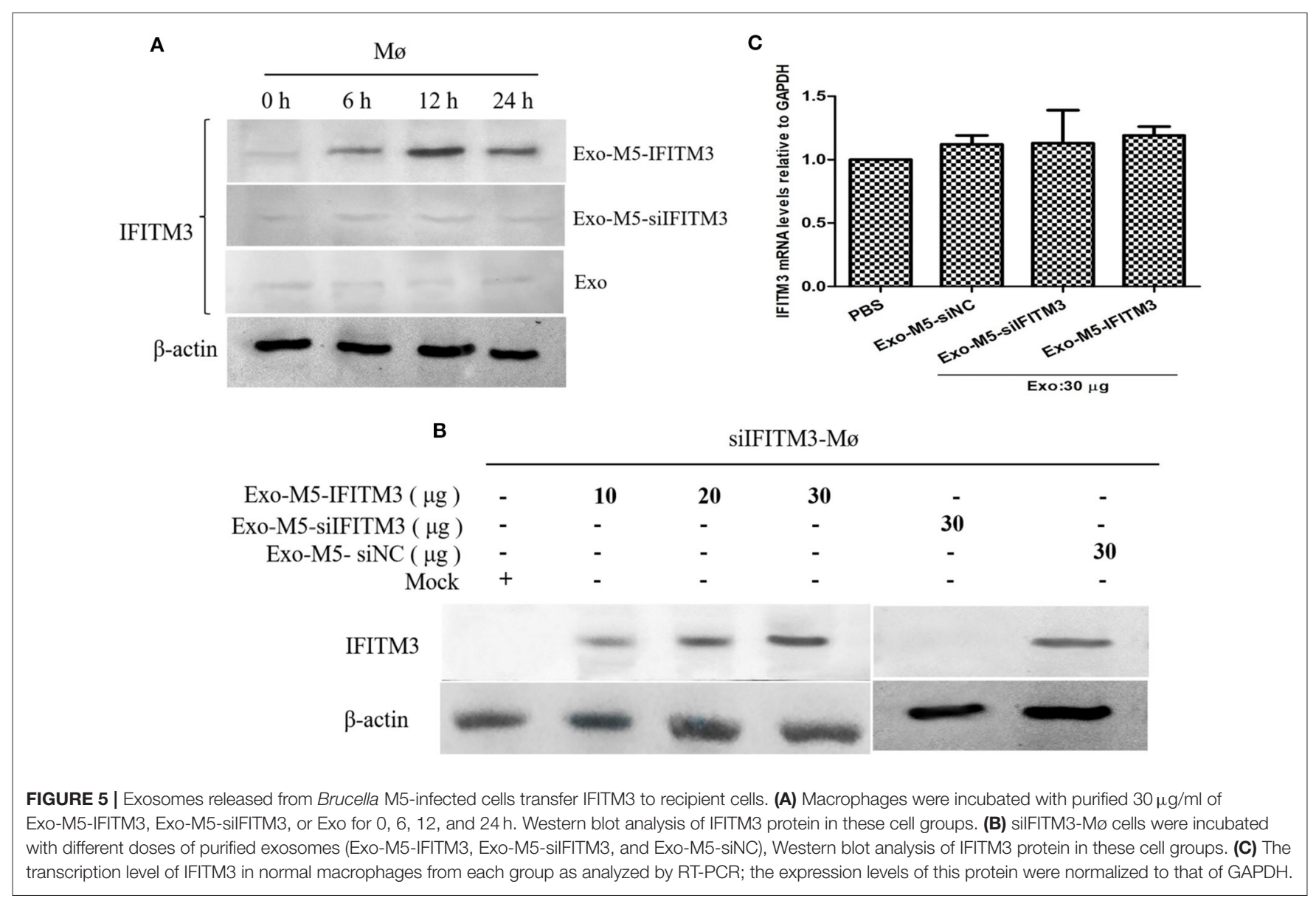




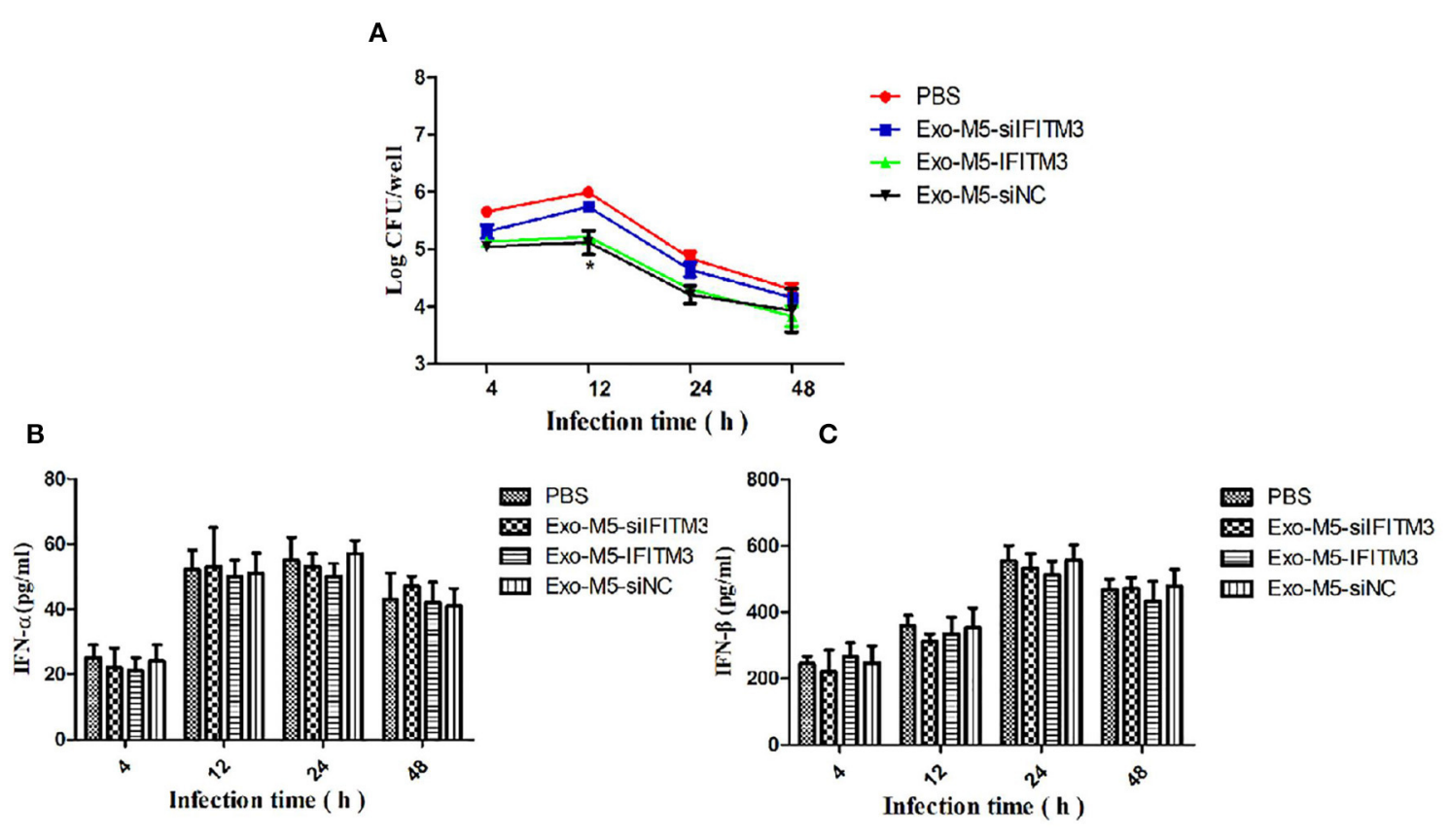

FIGURE 6 | The anti-Brucella activity of IFITM3-laden exosomes. siFITM3-Mø cells incubated with PBS, Exo-M5-silFITM3, Exo-M5-IFITM3, or Exo-M5-siNC were infected with Brucella M5. The CFU level was measured at different time points post-infection. (A) The intracellular bacterial survival was tested by measuring CFU, with PBS as a control group. The macrophage culture supernatants were harvested and IFN- $\alpha$ (B) and IFN- $\beta$ (C) production (pg/ml) were assessed using the ELISA assay. All treatments were repeated three times with $n=3 /$ time point. Statistical significance is indicated by ${ }^{*} p<0.05$.

A

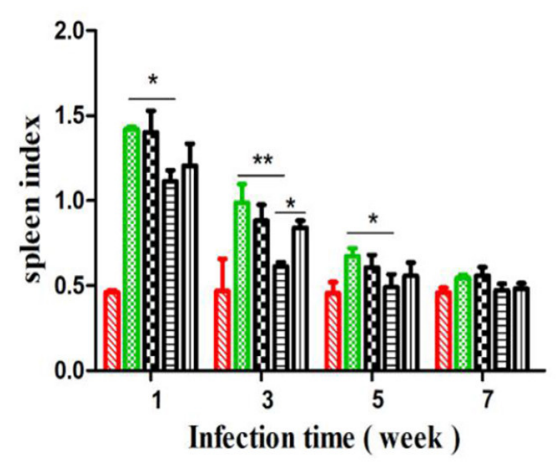

B

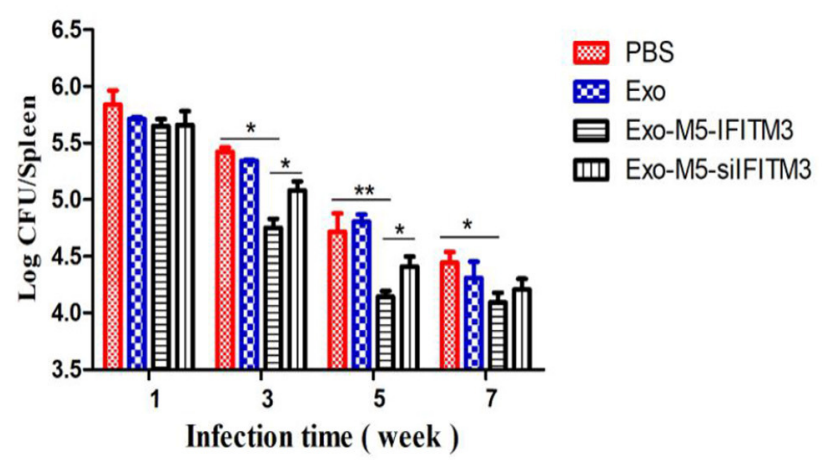

FIGURE 7 | Mice immunized with exosomes carrying IFITM3 were protected against Brucella infection. Mice immunized with different groups of exosomes were infected with Brucella M5 for 1, 3, 5, and 7 weeks infection. Then, each group of mice was sacrificed, and mice spleen index (A) and spleen CFU (B) were measured. All treatments were repeated three times with $n=3 /$ time point. Statistical significance is indicated by ${ }^{*} p<0.05,{ }^{* *} p<0.01$.

reproduction. Accordingly, the degree of activation of IFITM3 affected the efficiency of bacterial intracellular survival in the process of Brucella infection.

Exosomes are important mediators of intercellular information exchange. Exosomes can transfer proteins, nucleic acids, and lipids to neighboring or distant cells, and thus help to regulate various physiological or pathological processes (27). Recent studies have found that Mtb infection can induce immune cells to release exosomes containing bacterial antigens and related immune factors. These exosomes play an important role in initiating the host natural immunity and acquired immune response (28). In this study, we also successfully isolated and identified Brucella M5-infected macrophage-derived exosomes for the first time. In order to further determine the protein composition of the exosomes derived from Brucella-infected cells, we compared the proteomes of exosomes released from Brucella-infected (Bru-inf-Exo) and uninfected macrophages using label-free quantitation technology. We found that 89 proteins were increased in abundance in Bru-inf-Exo, including several proteins associated with immune function, such as IFITM3, Lyz2, and CD14. IFITM3 had the highest protein abundance. Interestingly, 
IFITMT-laden exosomes might be considered as novel mediators in the transfer of antiviral effects from infected cells to uninfected cells (19). Therefore, we tested whether the exosomes carrying IFITM3 could transfer the anti-Brucella activity to other uninfected cells. The results demonstrated that IFITM3 protein induced by Brucella infection was mainly released into the extracellular space in the form of exosomes, and the ability to release IFITM3 was related to the number of infecting Brucella. According to (29) during the formation of exosomes, IFITM3 can be transferred into the multivesicular complex (MVB). Rab family members are involved in the formation and release of exosomes carrying IFITM3 (29). However, a high concentration of Brucella, as discovered by our present study, may interfere with the biological function of macrophages; this would impede exosomes from loading and transporting the IFITM3 protein. Because of the special membrane structure of exosomes, they can fuse with receptor cell membranes. We demonstrated here that exosomes carrying IFITM3 could be internalized by receptor cells, thereby increasing the abundance of exogenous IFITM3 protein in recipient cells. This in turn can effectively inhibit the intracellular survival of Brucella M5. This immune pathway appears to represent a new, attractive potential anti-intracellular bacteria strategy.

Animal vaccination is still the most effective way to control the spread of brucellosis (30). As a special immune mediator, exosomes have been used in the development of many new vaccines. One study found that exosomes derived from macrophages infected with Mtb provide protective immunity to mice, suggesting that exosomes might serve as a new cell-free vaccine or immunopotentiator against Mtb infection. ISGs have been demonstrated to be attractive and efficient anti-microbial effectors (31). Accordingly, it is of great clinical significance to use exosomes as a kind of nano-shuttle carrier for immune factors or drug delivery (32). In such a situation, IFITM3laden exosomes may provide a direction for the development of new Brucella vaccines. It will be necessary to conduct small animal studies to test the feasibility and effectiveness of applying exosome-based IFITM3 delivery for anti-Brucella purposes in hosts. The spleen is the organ with the highest abundance of immune cells, and it is also the most active place for Brucella reproduction (33). Our results showed that IFITM3laden exosomes could more effectively reduce spleen damage and spleen CFU during the process of Brucella infection in mice compared with exosomes lacking IFITM3, confirming that IFITM3-containing exosomes may be one of the main treatments to improve the immune response and inhibit the proliferation of Brucella. Previous studies demonstrated that exosomes released from macrophages infected with Mycobacterium bovis stimulated host cell production of antigen-specific IFN- $\gamma$ and high levels of TNF- $\alpha$ and IL-12/p40 $(34,35)$. Consequently, further studies are

\section{REFERENCES}

1. Ahmed W, Zheng K, Liu ZF. Small non-coding RNAs: new insights in modulation of host immune response by intracellular bacterial pathogens. Front Immunol. (2016) 7:431-6. doi: 10.3389/fimmu.2016.00431 needed to explore how IFITM3-containing exosomes improve the immune level and thereby inhibit persistent infection of Brucella in vivo, and to determine whether there are other immune molecules and antigens in exosomes involved in the activation of anti-Brucella immunity in mice.

In summary, our data provide the first evidence for the existence of an extracellular vesicle, an exosome carrying IFITM3, which can transmit anti-Brucella activity from one cell to another, thereby effectively inhibiting Brucella infection in the host. This conclusion provides a theoretical foundation for the systematic elaboration of the mechanism of Brucella infection and host immune response, and our findings have provided new ideas for the development of potential candidate vaccines for Brucella.

\section{DATA AVAILABILITY STATEMENT}

The raw data supporting the conclusions of this article will be made available by the authors, without undue reservation.

\section{ETHICS STATEMENT}

The procedure of animal experiments was approved by the Institutional Animal Care and Use Committee of the First Affiliated Hospital of medical college, Shihezi Universityy (No. A201814901). Written informed consent was obtained from the owners for the participation of their animals in this study.

\section{AUTHOR CONTRIBUTIONS}

JY, YuW, and CC were responsible for designing experiments. JY, YoW, HZ, JX, and XD performed the experiments and assisted with the sample collections. JY and NY analyzed the data. JY, $\mathrm{YuW}$, and ZM wrote and revised the manuscript. All authors read and approved the final manuscript.

\section{FUNDING}

This work was supported by Open Fund of Key Laboratory of Control and Prevention of Animal Disease, Xinjiang Production \& Construction Corps (2020BTDJ02), the National Natural Science Foundation of China (No. U1803236), Research Projects of High-level Talents in Shihezi University (RCZK202040), National Natural Key Research and Development Plan (No. 2017YFD0500304), and Corps Major Scientific and Technological Projects (No. 2017AA003).

\section{ACKNOWLEDGMENTS}

We thank LetPub (www.letpub.com) for its linguistic assistance during the reparation of this manuscript.

2. Li ZQ, Dan G, Sun ZH, Zhang H, Gui D, Zhang JB, et al. Immunization of BALB/c mice with Brucella abortus $2308 \delta$ wbkA confers protection against wild-type infection. J Vet Sci. (2015) 16:467-73. doi: 10.4142/jvs.2015.16.4.467

3. Lacerda TLS, Cardoso PG, Almeida LAD. Inactivation of formyl transferase $(\mathrm{wbkC})$ gene generates a Brucella abortus rough strain 
that is attenuated in macrophages and in mice. Vaccine. (2010) 28:5627-34. doi: 10.1016/j.vaccine.2010.06.023

4. Martirosyan A, Moreno E, Gorvel JP. An evolutionary strategy for a stealthy intracellular Brucella pathogen. Immunol Rev. (2015) 240:211-34.

5. Zwerdling A, Delpino MV, Pasquevich KA. Brucella abortus activates human neutrophils. Microbes Infect. (2009) 11:68997. doi: 10.1016/j.micinf.2009.04.010

6. Lee WS, Shin JS, Jang DS, Lee KT. Cnidilide. An alkylphthalide isolated from the roots of Cnidium offici-nale, suppressesLPS-induced NO, PGE2, IL-1 $\beta$, IL- 6 and TNF- $\alpha$ production by AP-1 and NF- $\mathrm{B}$ inacti-vation in RAW 264.7 macrophages. Int Immunopharmacol. (2016) 40:14655. doi: 10.1016/j.intimp.2016.08.021

7. Osman AY, Abdullah FFJ, Kadir AA. Immuno-pathophysiological responses of mouse model to experimental infection with Brucella melitensis and its lipopolysaccharides via intraperitoneal route. Microb Pathog. (2016) 100:1770. doi: 10.1016/j.micpath.2016.08.019

8. Théry C, Ostrowski M, Segura E. Membrane vesicles as conveyors of immune responses. Nat Rev Immunol. (2009) 9:581-93. doi: 10.1038/nri2567

9. Mittelbrunn M, Sanchez-Madrid F. Intercellular communication: diverse structures for exchange of genetic information. Nat Rev Mol Cell Biol. (2012) 13:328-35. doi: 10.1038/nrm3335

10. Skog J, Würdinger T, Van RS. Glioblastoma microvesicles transport RNA and proteins that promote tumour growth and provide diagnostic biomarkers. Nat. Cell Biol. (2008) 10:1470-6. doi: 10.1038/ ncb 1800

11. Mittelbrunn M, Gutiérrez-Vázquez C, Villarroya-Beltri C. Unidirectional transfer of microRNA-loaded exosomes from $\mathrm{T}$ cells to antigen-presenting cells. Nat Commun. (2011) 2:282-8. doi: 10.1038/ncomms1285

12. Meckes DG, Shair KHY, Marquitz AR. Human tumor virus utilizes exosomes for intercellular communication. Proc Natl Acad Sci USA. (2010) 107:203705. doi: 10.1073/pnas.1014194107

13. Lee J, Kim SH, Choi DS, Lee JS, Kim DK, Go G, et al. Proteomic analysis of extracellular vesicles derived from Mycobacterium tuberculosis. Proteomics. (2015) 15:3331-7. doi: 10.1002/pmic.201500037

14. Mot YY, Othman I, Sharifah SH. Synergistic antibacterial effect of coadministering adipose-derived mesenchymal stromal cells and Ophiophagus hannah l-amino acid oxidase in a mouse model of methicillin-resistant Staphylococcus aureus-infected wounds. Stem Cell Res Ther. (2017) 8:58. doi: 10.1186/s13287-016-0457-2

15. Siegrist F, Ebeling M, Certa U. The small interferon-induced transmembrane genes and proteins. J Interf Cytok Res. (2011) 31:183-97. doi: 10.1089/jir.2010.0112

16. Wong MT, Chen SL. Emerging roles of interferon-stimulated genes in the innate immune response to hepatitis C virus infection. Cell Mol Immun. (2016) 13:11-35. doi: 10.1038/cmi.2014.127

17. Ranjbar S, Haridas V, Jasenosky L, Jasenosky LD, Falvo JV,Goldfeld AE. A role for IFITM proteins in restriction of mycobacterium tuberculosis infection. Cell Rep. (2015) 2015:874-74. doi: 10.1016/j.celrep.2015.09.048

18. Spence JS, He R, Hoffmann HH, Das T, Thinon E, Rice CM, et al. IFITM3 directly engages and shuttles incoming virus particles to lysosomes. Nat Chem Biol. (2019) 3:259-68. doi: 10.1038/s41589-0180213-2

19. Zhu X, He Z, Yuan J, Wen W, Huang X, Hu Y, et al. IFITM3containing exosome as a novel mediator for anti-viral response in dengue virus infection. Cell Microbiol. (2015) 17:105-18. doi: 10.1111/ cmi.12339

20. Mitchell MD. Exosome enrichment by ultracentrifugation and size exclusion chromatography. Front Biosci Landmrk. (2018) 23:865-65. doi: 10.2741/ 4621
21. Cheng Y, Schorey JS. Exosomes carrying mycobacterial antigens can protect mice against Mycobacterium tuberculosis infection. Eur J Immunol. (2013) 43:3279-27. doi: 10.1002/eji.201343727

22. Essandoh K, Yang L, Wang X, Huang W, Qin D, Hao J, et al. Blockade of exosome generation with GW4869 dampens the sepsis-induced inflammation and cardiac dysfunction. BBA Mol Basis Dis. (2015) 1852:236271. doi: 10.1016/j.bbadis.2015.08.010

23. Brass AL, Huang, IC, Benita Y, John SP, Krishnan MN, et al. The IFITM proteins mediate cellular resistance to influenzaAH1N1 virus, WestNile virus, and dengue virus. Cell. (2009) 139:1243-54. doi: 10.1016/j.cell.2009. 12.017

24. Gorman MJ, Poddar S, Farzan M, Diamond, MS. The interferon-stimulated gene Ifitm 3 restricts West Nile virus infection and pathogenesis. J Virol. (2016) 90:8212-25. doi: 10.1128/JVI.00581-16

25. Ranjbar SH, Viraga N, Aya J, Luke S, Supriya E, Thomas H, et al. Cytoplasmic RNA sensor pathways and nitazoxanide broadly inhibit intracellular Mycobacterium tuberculosis growth. iScience. (2019) 22:299313. doi: 10.1016/j.isci.2019.11.001

26. Smith LM, May RC. Mechanisms of microbial escape from phagocyte killing. Biochem Soc Trans. (2013) 41:475-90. doi: 10.1042/BST20130014

27. Schorey JS, Bhatnagar S. Exosome function:from tumor immunology to pathogen biology. Traffic. (2008) 9:87181. doi: 10.1111/j.1600-0854.2008.00734.X

28. Ramachandra L, Qu Y, Wang Y, Lewis CJ, Cobb BA, Takatsu K, et al. Mycobacterium tuberculosis synergizes with ATP To induce release of microvesicles and exosomes containing major histocompatibility complex class II molecules capable of antigen presentation. Infect Immun. (2010) 78:5116-25. doi: 10.1128/IAI.01089-09

29. Feeley EM, Sims JS, John SP, Chin CR, Pertel T, Chen LM. IFITM3 inhibits influenza A virusinfection by preventing cytosolic entry. PLoS Pathog. (2011) 7:e1002337. doi: 10.1371/journal.ppat.1002337

30. Simborio HLT, Reyes AWB, Hop HT, Arayan LT, Kim S. Strategies for the development of an effective vaccine against brucellosis. Prev Vet Med. (2014) 38:53-60. doi: 10.13041/jpvm.2014.38.2.53

31. Sadler AJ, Williams BR. Interferon-inducible antiviral effectors. Nat Rev Immunol. (2008) 8:559-68. doi: 10.1038/nri2314

32. Maryam K, Zahra S. Exosomes, microvesicles as diagnosis, therapeutic and drug delivery tools. international Pharmacy Acta. (2018) 1:100-1. doi: 10.22037/ipa.v1i1.19968

33. Mackaness BG. The immunological basis of acquired cellular resistance. J Exp Med. (1994) 120:105-20. doi: 10.1084/jem.120.1.105

34. Giri PK, Schorey JS. Exosomes derived from M. Bovis BCG infected macrophages activateantigen-specific $\mathrm{CD}^{+}$and $\mathrm{CD}^{+} \mathrm{T}$ cells in vitro and in vivo. PLoS ONE. (2008) 3:e246. doi: 10.1371/journal.pone.0002461

35. Beatty WL, Russell DG. Identification of mycobacterial surface proteins released into subcellular compartments of infected macrophages. Infect Immun. (2000) 68:6997-7002. doi: 10.1128/IAI.68.12.6997-7002.2000

Conflict of Interest: The authors declare that the research was conducted in the absence of any commercial or financial relationships that could be construed as a potential conflict of interest.

Copyright (c) 2021 Yi, Wang, Zhang, Deng, Xi, Li, Yang, Ma, Wang and Chen. This is an open-access article distributed under the terms of the Creative Commons Attribution License (CC BY). The use, distribution or reproduction in other forums is permitted, provided the original author(s) and the copyright owner(s) are credited and that the original publication in this journal is cited, in accordance with accepted academic practice. No use, distribution or reproduction is permitted which does not comply with these terms. 